\title{
CRISPR/Cas9-multiplexed editing of Chinese hamster ovary B4Gal-T1, 2, 3 and 4 Tailors N-Glycan Profiles of Therapeutics and Secreted Host Cell Proteins
}

Amann, Thomas; Hansen, Anders Holmgaard; Kol, Stefan; Min Lee, Gyun; Andersen, Mikael Rørdam; Kildegaard, Helene Faustrup

\section{Published in:}

Biotechnology Journal

Link to article, DOI:

10.1002/biot.201800111

Publication date:

2018

Document Version

Peer reviewed version

Link back to DTU Orbit

Citation (APA):

Amann, T., Hansen, A. H., Kol, S., Min Lee, G., Andersen, M. R., \& Kildegaard, H. F. (2018). CRISPR/Cas9multiplexed editing of Chinese hamster ovary B4Gal-T1, 2, 3 and 4 Tailors $N$-Glycan Profiles of Therapeutics and Secreted Host Cell Proteins. Biotechnology Journal, 13(10), [1800111 ].

https://doi.org/10.1002/biot.201800111

\section{General rights}

Copyright and moral rights for the publications made accessible in the public portal are retained by the authors and/or other copyright owners and it is a condition of accessing publications that users recognise and abide by the legal requirements associated with these rights.

- Users may download and print one copy of any publication from the public portal for the purpose of private study or research.

- You may not further distribute the material or use it for any profit-making activity or commercial gain

- You may freely distribute the URL identifying the publication in the public portal 
CRISPR/Cas9-multiplexed editing of Chinese hamster ovary B4Gal-T1, 2, 3 and 4 tailors N-glycan profiles of therapeutics and secreted host cell proteins

Thomas Amann ${ }^{1 *}$, Anders Holmgaard Hansen ${ }^{*}$, Stefan Kol ${ }^{1}$, Gyun Min Lee ${ }^{1,2}$, Mikael Rørdam Andersen $^{3}$, Helene Faustrup Kildegaard ${ }^{1}$

${ }^{1}$ Novo Nordisk Foundation Center for Biosustainability, Technical University of Denmark, Kgs. Lyngby, Denmark

2Department of Biological Sciences, KAIST, Daejeon, Republic of Korea 3Department of Biotechnology and Biomedicine, Technical University of Denmark, Kgs. Lyngby, Denmark

*These authors contributed equally to this publication

Correspondence: Helene Faustrup Kildegaard, Novo Nordisk Foundation Center for Biosustainability, Kemitorvet, Building 220, 2800 Kgs. Lyngby, Denmark

E-mail: hef@biosustain.dtu.dk

Keywords: Chinese hamster ovary cells, CRISPR/Cas9, N-glycosylation, Glycoengineering, Multiplexing, Rituximab, Erythropoietin

\section{Abbreviations:}

Asn, Asparagine; AUC, area under curve; B4Gal-T, $\beta$-1,4-galactosyltransferase; Cas9, CRISPR-associated protein 9; CHO, Chinese hamster ovary; CRISPR, clustered regularly interspaced short palindromic repeats; EPO, erythropoietin; FACS, fluorescence-activated cell sorting; Fc, fragment crystallizable; Fc $\boldsymbol{\gamma}$ RIIIa, Fc-gamma 
receptor III a; FUT8, alpha-(1,6)-fucosyltransferase; G0, agalactosylated; GIcNAc, N-Acetylglucosamine; HM, high-mannose; HPC4, human protein C4; IgG, immunoglobulin G; indel, insertion or deletion; mAb, monoclonal antibody; sgRNA, single guide RNA; TSTA3, tissue-specific transplantation antigen P35B; UDP-Gal, uridine diphosphate galactose; VCD, viable cell density; WT, wildtype

\begin{abstract}
In production of recombinant proteins for biopharmaceuticals, N-glycosylation is often important for protein efficacy and patient safety. IgG with agalactosylated (G0)-N-glycans can improve the activation of the complement system and be advantageous in the therapy of lupus and virus diseases. In this study, we aimed to engineer CHO-S cells for the production of proteins with G0-N-glycans by targeting B4Gal-T isoform genes with CRISPR/Cas9. Indel mutations in genes encoding B4Gal-T1, -T2, -T3 with and without disrupted B4Gal-T4 sequence resulted in only $\sim 1 \%$ galactosylated N-glycans on total secreted protein of three clones per genotype. In the triple-KO clones, transiently expressed erythropoietin (EPO) and transiently expressed rituximab harbored only $\sim 6 \%$ and $\sim 3 \%$ galactosylated N-glycans, respectively. However, simultaneous disruption of B4Gal-T1, -T2 and -T3 was found to decrease cell growth. We furthermore revealed possible B4Gal-T isoform branch preferences where B4Gal-T2 activity is restricted to act on a single $\mathrm{N}$-glycan branch and B4Gal-T4 was found to be inactive in N-glycan galactosylation in CHO-S cells. Altogether, we present the advantage of analyzing total secreted protein N-glycans after disrupting glycosyltransferases followed by expressing recombinant proteins in selected clones with desired N-glycan profiles at a later stage. Furthermore, we provide a cell platform that prevalently glycosylates proteins with G0-N-glycans to further study the impact of agalactosylation on different in vitro and in vivo functions of recombinant proteins.
\end{abstract}




\section{Introduction}

Chinese hamster ovary (CHO)-derived cells are the major workhorses within mammalian cell lines and represent the cell platform in which $>50 \%$ of the marketed recombinant proteins are produced[1]. Thereof, recombinant monoclonal antibodies (mAbs) are the main product subclass and are utilized for the treatment of cancer and various inflammatory diseases[2]. As a result of post-translational protein processing, mAbs harbor two N-glycans, one on each heavy chain at Asparagine (Asn) 297 whereas erythropoietin (EPO) has three N-glycosylation sites occupied by predominantly tri- and tetra-antennary structures [3]. In general, N-glycosylation can impact protein folding, immune regulation, cellular homeostasis and the biological half-life of proteins $[4,5]$. Within mAbs, the fragment crystallizable (Fc) N-glycans at Asn297 have a strong influence on anti-inflammatory properties, antibody-dependent cell-mediated cytotoxicity and complement-dependent cytotoxicity[6].

The heterogeneous $\mathrm{N}$-glycan profile of glycoproteins produced in $\mathrm{CHO}$ is one of the main factors that cause mAb heterogeneity and can be further optimized regarding corefucosylation, galactosylation, antennarity and terminal capping by sialic acids. Rituximab is an immunoglobulin G (IgG) 1-class molecule, one of the recombinant glycoproteins produced in CHO, and exceeds annual revenues of USD 7 billion[7]. Rituximab targets the B-cell surface antigen CD20 in B-cell lymphoma and is predominantly N-glycosylated by A2FG0 and A2FG1 structures when produced in non-glyco-engineered CHO cells[8]. Since several studies revealed non-fucosylated IgGs have significantly higher binding affinity for the Fc-gamma receptor IIIa (FcyRIIIa) than fucosylated $\operatorname{IgG}$ versions[9, 10], different approaches successfully removed the core-fucose by knockout of alpha- $(1,6)$ fucosyltransferase (FUT8) or tissue specific transplantation antigen P35B (TSTA3) in IgGexpressing CHO cell lines[11-14]. 
Additionally, agalactosylated IgG1 variants with terminal N-Acetylglucosamine (GlcNAc) (referred to as G0 glycoforms) can increase the binding to FcRIIIa[15] and are accessible for the mannose-binding protein. They can therefore promote activation of the complement system[16] without impacting in vivo clearance[17-19]. Furthermore, HIV patients with high viral inhibition displayed an increased proportion of agalactosylated N-glycans on global serum IgG, suggesting that agalactosylated IgG variants may have antiviral activity[20]. Interestingly, Lupus patients showed improved disease symptoms after treatment with agalactosylated antibodies[21]. These G0-IgG variants can be obtained by sequential treatment of wild type (WT)-IgG with neuraminidase and galactosidase or supplementing cultivation medium with galactose analogues to block cellular B4Gal-Ts[22]. Nevertheless, fewer cell-engineering attempts were initiated to produce G0-IgG1 compared to engineering non-fucosylated IgG1 variants.

Since the CHO genome sequence is publically available[23], CHO cell-engineering is no longer performed in a "black box", which shortens cell line development and empowers a targeted approach for the engineering of a G0 $\mathrm{CHO}$ cell line. The classes of glycosyltransferases are made of homologous gene families, where the class of $\beta-1,4$ galactosyltransferases (B4GalT) consists of seven members, B4Gal-T1-T7, which all transfer galactose from uridine diphosphate galactose (UDP-Gal) to GlcNAc and GlcNActerminated oligosaccharides (EC 2.4.1.38)[24, 25]. The seven CHO B4Gal-Ts all share a common WGXEDD sequence as part of their B4Gal-T motif[26] and have exclusive specificity for the donor substrate UDP-Gal. B4Gal-T5 and -T6 are described to mainly function in 0-glycosylation[27, 28], whereas B4Gal-T7 transfers UDP-Gal within glycosaminoglycan biosynthesis and therefore is not involved in the $\mathrm{N}$-glycosylation of proteins $[29,30]$. A further study indicated that B4Gal-T1, -T2, -T3 and -T4 perform Nglycan galactosylation more efficient than B4Gal-T5 and -T6 and suggested different branch preferences for the family members of $\beta$-1,4-galactosyltransferases[31]. In addition, B4Gal- 
T4 is reported to also be active in the galactosylation of mucin-type core 2 branching in the O-glycosylation pathway[32]. Another study described B4Gal-T1-KO mutants to have dramatically reduced galactosylation on secreted host cell protein (secretome) N-glycans and reduced growth of mice[28, 33]. In a previous study performed with CHO-K1 derived cell lines, triple-KO of B4Gal-T1, -T2 and -T3, double-KO of B4Gal-T1 and -T3 and single-KO of B4Gal-T1 led to almost fully agalactosylated EPO and rituximab[34]. However, that study was based on only one clone per KO-combination and therefore did not consider clonal variation and did not investigate the impact of B4Gal-T disruptions on cell growth. Although this suggests $\mathrm{B} 4 \mathrm{Gal}-\mathrm{T} 1$ and $-\mathrm{T} 3$ to be the main players in galactosylation of rituximab and EPO N-glycans in CHO-K1 cells, the N-glycosylation activity of B4Gal-T1, -T2, -T3 and -T4, specifically in the industrially relevant CHO-S cell line, needs to be further explored to generate a fully G0 cell line within CHO-S cells. Since the effect of single disruptions of B4GalT1, -T2, -T3 and -T4 on N-glycosylation was investigated in previous work[34], we designed a multiplexing approach to disrupt combinations of up to four B4Gal-Ts. The target design for the multiplexing approach contained B4Gal-T1, B4Gal-T3 or both targets in combination with B4Gal-T2 and/or B4Gal-T4. With the help of multiplexing, the effect of stacking B4GalT disruptions could be studied with regards to cell growth and protein N-glycosylation on three clones for each triple- and quadruple-KO combination to additionally examine clonal variation.

In this work, we used clustered regularly interspaced short palindromic repeats/CRISPRassociated protein 9 (CRISPR/Cas9) as a tool for multiplexed editing of gene targets within the same transfection[13]. B4Gal-T1 and -T3 as well as B4Gal-T2 and -T4 were disrupted simultaneously to facilitate investigation of the combinatorial effect of B4Gal-T knockouts on N-glycosylation and cell growth. N-glycosylation analysis of total secreted proteins, as well as transiently expressed rituximab and EPO (representing dissimilar N-glycan profiles), in B4Gal-T edited CHO-S cell lines was performed. The analysis demonstrates that 
$\mathrm{N}$-glycans can be tailored for a greater variety of secreted glycoproteins, as represented by more than 250 proteins within the CHO-S secretome[35] in addition to EPO and rituximab. With this, we investigated if screening the secretome $\mathrm{N}$-glycans of our engineered clones is a promising strategy towards the expression of rituximab and EPO with G0 N-glycans in selected clones. The strategy was built on (i) analyzing the N-glycan profile of all secreted proteins from selected multiplexed clones to then (ii) expressing rituximab and EPO in selected clones for rituximab/EPO N-glycan analysis and (iii) evaluating of the role of each targeted B4Gal-T within the galactosylation of N-glycans. Especially the role of B4Gal-T2 and -T4 in CHO-S and the effect of B4Gal-T indels on cell growth, both with respect to clonal variation, have to our knowledge not been investigated previously and were the driving motives of this work.

\section{Materials and methods}

\section{1. sgRNA and GFP_2A_Cas9 plasmid design}

GFP_2A_Cas9 and single guide RNA (sgRNA) plasmids were constructed as previously described [13]. The sgRNA target design for B4Gal-T1, B4Gal-T2, B4Gal-T3 and B4Gal-T4 was performed using CRISPy[36]. The target sites for the mentioned genes and the oligos for sgRNA cloning are listed in Supporting Information, Table S1 and Table S2, respectively.

\subsection{Cell cultivation and transfection for multiplexed genome editing}

CHO-S suspension cells (Life Technologies, Carlsbad, CA) were cultivated in CD CHO medium supplemented with $8 \mathrm{mM} \mathrm{L-glutamine} \mathrm{and} 1 \mu \mathrm{L} / \mathrm{mL}$ anti-clumping agent (Life Technologies). Cells were incubated in a humidified incubator at $120 \mathrm{rpm}, 37^{\circ} \mathrm{C}$ and $5 \% \mathrm{CO}_{2}$. Cell passaging was conducted every two to three days at $3 \times 10^{5}$ cells $/ \mathrm{mL}$ after measuring viable cell densities (VCDs) and viabilities with the NucleoCounter NC-200 Cell Counter (ChemoMetec, Allerod, Denmark). One day prior transfection with CRISPR reagents, anti- 
clumping agent was removed by centrifugation and $5-6 \times 10^{5}$ cells $/ \mathrm{mL}$ were seeded in a six multi-well well plate (BD Biosciences, San Jose, CA) for each transfection. At the day of transfection each sample was seeded at $1 \times 10^{6}$ cells/mL and a total DNA load of $3.5 \mu \mathrm{g}$ was transfected with FuGENE ${ }^{\circledR}$ HD transfection reagent (Promega, Madison, WI) and OptiPRO SFM medium (Life Technologies) according to the manufacturer's recommendations. The GFP_2A_Cas9 / sgRNA plasmid ratios for each sample are presented in Table S3. To measure transfection efficiency, pmaxGFP ${ }^{\circledR}$ vector (Lonza, Basel, Switzerland) transfection was performed. Cells were harvested for fluorescence-activated cell sorting (FACS) $48 \mathrm{~h}$ after transfection.

\subsection{Single cell cloning using FACS}

Before FACS, cells were filtered through a $40 \mu \mathrm{m}$ cell strainer into a FACS-compatible tube. Operating a FACSJazz (BD Biosciences) single fluorescent-positive cells were sorted into 384-well plates (Corning, New York, NY) already containing $30 \mu \mathrm{L}$ CD CHO medium supplemented with $8 \mathrm{mM}$ L-glutamine, 1.5\% HEPES buffer and 1\% Antibiotic-Antimycotic (Gibco, Waltham, MA) per well. For cell sorting, fluorescent-positive cell populations were gated based on non-transfected WT CHO-S cells. Two weeks after cell sorting the clones were moved to 96-well flat-bottom plates (BD Biosciences) and expanded for deep sequencing analysis and batch cultivation.

\subsection{Deep sequencing analysis}

Confluent colonies from 96-well flat-bottom replicate plates were harvested for genomic DNA extraction. DNA extraction was performed using QuickExtract DNA extraction solution (Epicentre, Illumina, Madison, WI) according to the manufacturer's instruction. The library preparation was based on Illumina 16S Metagenomic Sequencing Library Preparation and deep sequencing was carried out on a MiSeq Benchtop Sequencer (Illumina, San Diego, CA). 
The protocol for amplifying the targeted genomic sequences, amplicon purification, adapter-PCR and following quality analysis was based on previously published work [13]. PCR primers are presented in Supporting information, Table S4.

\subsection{Batch cultivation to study cell growth and secretome $\mathbf{N}$-glycans}

For batch cultivation and secretome analysis, cells were seeded at $3.0 \times 10^{5}$ cells $/ \mathrm{mL}$ in Corning vent cap shake flasks (Sigma-Aldrich, St. Louis, MI) as duplicates in $30 \mathrm{~mL}$ CD CHO medium supplemented with $8 \mathrm{mM} \mathrm{L}$-glutamine and $1 \mu \mathrm{L} / \mathrm{mL}$ anti-clumping agent (Life Technologies). Cells were incubated in a humidified incubator at $120 \mathrm{rpm}, 37^{\circ} \mathrm{C}$ and $5 \% \mathrm{CO}_{2}$. Cell densities and viabilities were determined once per day using the NucleoCounter NC250 Cell Counter (ChemoMetec). Secretome sample volume was calculated to harbor $20 \mathrm{x}$ $10^{6}$ cells and harvested five days after seeding to be pooled within biological replicates.

\subsection{Batch cultivation for transient rituximab/EPO transfection and rituximab/EPO}

\section{$\mathrm{N}$-glycan analysis}

For transient expression of rituximab/EPO, cells were seeded in Corning vent cap shake flasks (Sigma-Aldrich) as duplicates with cell densities $\sim 1 \times 10^{6}$ cells/mL in $60 \mathrm{~mL}$ CD CHO medium supplemented with $8 \mathrm{mM}$ L-glutamine (Life Technologies). Cells were incubated in a humidified incubator at $120 \mathrm{rpm}, 37^{\circ} \mathrm{C}$ and $5 \% \mathrm{CO}_{2}$ and transfected with $75 \mu \mathrm{g}$ of rituximab or EPO encoding plasmid for each flask using FreeStyleTM MAX reagent together with OptiPRO SFM medium (Life Technologies) according to the manufacturer's recommendations. $1 \mu \mathrm{L} / \mathrm{mL}$ anti-clumping agent was added $24 \mathrm{~h}$ after transfection. pmaxGFP ${ }^{\circledR}$ vector (Lonza) transfection was performed to measure transfection efficiencies. Cell densities and viabilities were determined once per day using the NucleoCounter NC250 Cell Counter (ChemoMetec). To purify rituximab and EPO, the supernatants of the 
transfected clones were harvested three days after transfection and pooled within duplicates.

\subsection{Rituximab and EPO purification}

For rituximab purification, supernatant samples were centrifuged $\left(1000 \mathrm{~g}, 5\right.$ minutes, $\left.4^{\circ} \mathrm{C}\right)$ and afterwards filtered $(\sim 0.22 \mu \mathrm{m}$ pore size $)$ to remove cells and cell debris. Rituximab was purified by protein A affinity chromatography (MabSelect, GE Healthcare, Uppsala, Sweden) according to the manufacturer's protocol. Human protein C4 (HPC4)-tagged EPO was purified from supernatants using Anti-Protein C Affinity Matrix from Roche (Basel, Switzerland, Cat. Nr. 11815024001) as per the instructions of the manufacturer.

\subsection{N-Glycan analysis}

Sample preparation for N-glycan analysis was performed with GlycoWorks RapiFluor-MS N-Glycan Kit (Waters, Milford, MA) according to the manufacturer's instruction. $12 \mu \mathrm{g}$ purified protein or $12 \mu \mathrm{l}$ of $10 \mathrm{x}$ concentrated (Amicon Ultra-15, Merck, Darmstadt, Germany) secretome sample were used for each sample. Labeled N-Glycans were analyzed by a LC-MS system using a Thermo Ultimate 3000 HPLC with fluorescence detector coupled on-line to a Thermo Velos Pro Iontrap MS, as described previously with minor modifications [13]. Separation gradient $30 \%$ to $43 \%$ buffer and MS was run in positive mode. Amount of $\mathrm{N}$-Glycan was measured by integrating the areas under the normalized fluorescence spectrum peaks with Thermo Xcalibur software (Thermo Fisher Scientific, Waltham, MA) giving the normalized, relative amount of the glycans.

\section{Results}




\subsection{Generation of engineered $\mathrm{CHO}-\mathrm{S}$ cell lines with combinations of indels in multiple}

\section{B4Gal-T genes}

To investigate the exact impact of B4Gal-T1, -T2, -T3 and -T4-KO on N-glycan galactosylation, we aimed to generate clones with insertion or deletion (indel) mutations in one or several of the genes. To get these combinations in a minimal number of operations, we co-transfected Cas9 (GFP_2A_Cas9) with either sgRNAs against B4Gal-T1 and -T3 or against B4Gal-T1, -T2 and -T3 or sgRNAs against B4Gal-T1 and -T2 in the same transfection (Supporting Information, Table S3). After transfection and single cell cloning, we carried out deep sequencing to identify the genomic changes in the targeted sequences. We aimed to identify clones with exclusively out-of frame indels in one or more of the target sequences leading to a potentially functional knockout of the targeted glycosyltransferase(s) to investigate the effect on $\mathrm{N}$-glycan galactosylation. In a second round of transfections, we aimed to generate clones with indels in combinations of three or all four targeted B4Gal-Ts. Therefore we co-transfected GFP_2A_Cas9 with either sgRNAs against B4Gal-T1 and -T4 or against B4Gal-T1 into a clone with confirmed indels in B4Gal-T2 and -T3 (Table 1).

In our study, a total of 109 potential deletion clones were deep sequenced for genomic indels in the targeted regions (Supporting Information, Table S5). Out of these, 23 clones revealed an unclear genotype for one or more targets (presence of in-frame indel or indelfrequency between 5-98\%). These were discarded. We expanded clear single- and multi-KO clones of 1-4 targets (indel frequencies $>98 \%$ ). Next, we isolated multiple independent clones for each genotype to study true biological replicates of the phenotypes, in total 17 clones (Table 1). One clone (WT ctr) which showed no insertion or deletion was additionally selected to serve as a control for the analysis of growth and N-glycan profiles. A second clone (T2-3-KO ctr) which did not reveal additional indels after the second round of transfection was also characterized to investigate the impact of transfection and subcloning on growth and $\mathrm{N}$-glycan profile. 


\subsection{Effect on growth from different B4Gal-T-KO's and indel combinations}

The aim of our study is to provide a $\mathrm{CHO}$ platform to produce recombinant proteins with agalactosylated N-glycans. Through engineering cells towards G0-glycans, the N-glycans are altered not only on the recombinant protein, but also on host cell proteins. As cell growth performance is a substantial factor for industrial protein production platforms, we first evaluated whether decreased N-glycan galactosylation influence $\mathrm{CHO}$ cell growth. We carried out shake flask batch experiments with selected KO clones, the parental CHO-S WT as well as two control clones (Table 1). Sampling for VCDs and viabilities was performed every $24 \mathrm{~h}$ for the time course of seven days. The WT ctr clone was identified to have similar growth and viability to CHO-S WT (Fig. 1). Double-KO of B4Gal-T1 and -T3 (T1-3-KO) indicated slightly decreased growth compared to CHO-S WT and WT ctr (Fig. 1A). The two clones with frame-shifts in B4Gal-T3 (T3-KO A \& T3-KO B) were not influenced in cell growth and reached slightly higher maximal VCDs than CHO-S WT (Fig. 1A). Furthermore, the double-KO clone with indels in B4Gal-T2 and -T3 (T2-3-KO) revealed growth comparable to CHO-S WT and WT ctr (Fig. 1B). The T2-3-KO ctr clone exhibited lower growth compared to the growth curve of the parental T2-3-KO clone (Fig. 1B). The four triple-KO clones with frame-shifts in B4Gal-T1, -T2 and -T3 (T1-2-3-KO) and the three T12-3-4-KO mutants had decreased growth compared to CHO-S WT (Fig. 1B, Fig. 1C). The three T2-3-4-KO clones had heterogeneous growth and compared to CHO-S WT, similar maximal VCD (T2-3-4-KO C), lower maximal VCD (T2-3-4-KO K) or increased maximal VCD (T2-3-4-KO H) was observed (Fig. 1D). Lastly, the three T1-2-KO clones exhibited also heterogeneous growth but clone T1-2-KO B reached similar maximal viable cell densities compared to CHO-S WT (Fig. 1E).

Altogether, the engineered clones revealed a notable variation in growth within groups of clones with indels in the same target genes. Whereas T1-2-3-KO and T1-2-3-4-KO clones 
had decreased growth compared to CHO-S WT, clones with other indel combinations could grow to similar maximal viable cell densities as CHO-S WT.

\subsection{Effects of B4Gal-T-KO’s on secretome N-glycan profiles}

To investigate the activities of the targeted $\beta$-1,4-galactosyltransferases within protein $\mathrm{N}$ glycosylation, we analyzed secretome samples of CHO-S WT, the control clones and the different KO-clones with indels in 1-4 sequences for the targeted B4Gal-T-genes (Table 1). To examine the contribution of the targeted B4Gal-Ts within galactosylation of the different $\mathrm{N}$-glycan branches, we studied the remaining levels of $\mathrm{N}$-glycan galactosylation in clones with combinatorial disruption of B4Gal-Ts. To probe the effect of the generated indels on the secretome N-glycans of the selected clones, we analyzed supernatant samples harvested five days after seeding. Secretome samples were centrifuged and filtered to remove cells and cell debris and up-concentrated before total N-glycans were labeled and analyzed by HPLC/MS. As presented in supplementary Figure 1, the complex bi-antennary di-sialylated $\mathrm{N}$-glycan structure (A2FG2S2) was the major structure within the CHO-S WT secretome. Notably, in the CHO-S WT secretome, only one minor peak (0.7\%) of G0-N-glycan could be annotated (supplementary Fig. 1). T3-KO, T2-3-KO, and T2-3-4-KO clones showed a similar N-glycan pattern to WT but G0 structures were only present in T2-3-KO and T2-3-4-KO clones (Fig. 2). However, within the annotated N-glycan structures, the T1-3-KO clone exhibited a total of $\sim 65 \%$ G0 structures, but still displayed $\sim 10 \%$ mono-galactosylated Nglycans in the secretome (Fig. 2). Compared to CHO-S WT, indels in B4Gal-T1 and -T2 resulted in the absence of G4 forms, reduced G3 and G2 forms and increased G1 and G0 proportions (Fig. 2) leading to increased overall heterogeneity in N-glycan galactosylation (supplementary Fig. 1). T1-2-KO clones overall showed decreased galactosylated N-glycans compared to WT ( $\sim 61 \%$ galactosylated structures) and revealed $\sim 24 \%$ galactosylated structures. 
In contrast, we could only annotate $\sim 1 \%$ galactosylated $\mathrm{N}$-glycan structures in the secretomes of T1-2-3-KO and T1-2-3-4-KO clones (Fig. 2). The major N-glycan structures of T1-2-3-KO and T1-2-3-4-KO clones were A2FG0, A3FG0 and A4FG0. The bi-galactosylated structures, which were the predominant N-glycans in CHO-S WT and WT ctr clone, were not present anymore (see Fig. 2 and supplementary Fig. 1). Furthermore, the additional B4GalT4 indel in T1-2-3-4-K0 clones did not increase G0 proportions or eliminate G1 N-glycans when compared to T1-2-3-KO cell lines (Fig. 2).

Altogether, disruption of B4Gal-T2 in conjunction with B4Gal-T1 and -T3 decreased the galactosylated secretome N-glycan proportion from 10\% (T1-3-K0) down to 1\% (T1-23-KOs) and the secretome N-glycans of the triple- and quadruple-KOs were dominated by agalactosylated bi-, tri- and tetra-antennary structures with A2FG0 as the dominating Nglycan structure (supplementary Fig. 1).

To examine the role of the four targeted B4Gal-Ts within protein N-glycan galactosylation, we studied the levels of agalactosylation in the secretome samples in a comparative approach (Fig. 2). Confirming that B4Gal-T1 is the most active N-glycan $\beta-1,4$ galactosyltransferase, we furthermore studied the presence of agalactosylation without (clone T3-KO A and T1-3-KO) and with additional KO of B4Gal-T2 (clones T1-2-3-KO and T2-3-KO) to investigate its contributing role in N-glycan galactosylation, which has not previously been studied in exact terms. Therefore, we compared two sets of two clones, differing in their genotype by the KO of B4Gal-T2. In the first comparison, the single KO of B4Gal-T3 exhibited no G0-N-glycans, where the double-KO of B4Gal-T2 and -T3 revealed $\sim 6 \%$ G0-N-glycans (Fig. 2). Similarly, comparing the N-glycan proportion with terminal GlcNAc of clone T1-3-KO and T1-2-3-KO clones, the additional KO of B4Gal-T2 in the tripleKO cell lines increased the G0-N-glycan proportion by 10\%. 


\subsection{Tailored Rituximab and EPO N-glycosylation after B4Gal-T-double and triple- KO's}

To prove that engineered secretome N-glycans will also be represented on rituximab and EPO which we used as model proteins, we transfected a rituximab- or EPO-encoding plasmid into CHO-S WT and KO-clones T3-KO A, T2-3-KO, T1-3-KO and T1-2-3-KO. Cells were transfected and rituximab- or EPO-containing supernatants were harvested after three days. After purification, we analyzed the corresponding N-glycan structures within the different KO cell lines.

Clones T1-3-KO and T1-2-3-KO showed predominantly G0-N-glycans in the secretome samples and were expected to also reveal predominantly G0 structures on the transfected rituximab. CHO-S WT, clone T3-KO A and T2-3-KO displayed comparable rituximab Nglycan profiles with G0 and G1 as prevalent structures with both $\sim 40 \%$ of total rituximab N-glycans (Fig. 3A). In contrast, rituximab purified from clones T1-2-3-KO and T1-3-KO clones was mostly N-glycosylated by bi-antennary G0 structures, whereas G2 structures were missing, which is in line with secretome N-glycans of these clones. Notably, double-KO of B4Gal-T1 and -T3 in T1-3-K0 resulted in higher G0-N-glycan proportions on rituximab $(\sim 84 \%)$ than in clone T1-2-3-KO ( $68 \%)$. Furthermore, triple-KO clone T1-2-3-KO had increased high-mannose (HM) structures on rituximab when compared to the other cell lines.

Figure 3B presents a detailed comparison of rituximab N-glycans purified from T1-3-KO and T1-2-3-KO A. Here the bi-antennary, non-galactosylated A2FG0 was clearly the main structure. However, we could also annotate HM, A2G0 and A2FG1 N-glycans. Thereof A2FG1 was found in both clones to comparable amounts $(\sim 2-3 \%)$ but total HM proportions were higher in T1-2-3-KO (15\%) than in T1-3-KO (5.8\%), represented by Man5, Man7, Man8 and Man9 structures. Additionally, cell growth after rituximab transfection was comparable 
between CHO-S WT, WT ctr, T3-KO A and T1-3-KO (supplementary Fig. 2) whereas clones T2-3-KO and T1-2-3-KO revealed increased viable cell concentrations on day three.

For transiently expressed EPO, the N-glycan profiles of CHO-S WT, T3-KO A and T2-3-KO are similar where annotated $\mathrm{N}$-glycan structures predominantly harbor $\geq 4$ galactose residues; however G0 forms are not present in EPO from CHO-S WT (Fig. 3C). In contrast, double-KO of B4Gal-T1 and -T3 resulted in increased G0 proportions ( $72 \%)$ whereas G3and G4-glycans could not be identified on EPO purified from T1-3-KO. Analyzing N-glycan structures of EPO from the triple-KO clone T1-2-3-KO A, we could only annotate agalactosylated and mono-galactosylated N-glycans (supplementary Fig. 3).

Overall, disruption of B4Gal-T1 and -T3 with or without additional disruption of B4Gal-T2 resulted in rituximab, which only harbored $\sim 2-3 \%$ galactosylated N-glycans. On the other hand, single disruption of B4Gal-T3 or disruption of both B4Gal-T2 and -T3, did not change rituximab N-glycosylation compared to CHO-S WT (Fig. 3A). However, disruption of B4Gal$\mathrm{T} 2$ in addition to indels in B4Gal-T1 and -T3 increased the G0 N-glycan proportion of transiently expressed EPO from $\sim 72 \%$ to 91\% (Fig. 3C).

\section{Discussion}

Since recombinant proteins with agalactosylated N-glycans can be of interest for the therapy of several diseases, we aimed to engineer CHO-S cells to reveal predominantly agalactosylated N-glycans on secreted proteins and on transiently expressed rituximab and EPO. In a previous study in CHO-K1 derived cell lines, disruption of B4Gal-T1, -T2 and -T3 resulted in prevalently agalactosylated N-glycans on rituximab and EPO whereas the effects of B4Gal-T disruptions on cell growth and the glycosylation of total secreted proteins were not addressed[34]. Within this work, we also aimed to assess the impact of B4Gal-T indels on cell growth and analyze N-glycans and cell growth in groups of clones with the same combination of indels with respect to clonal variation. We investigated if disruption of 
B4Gal-T1, -T2 and -T3 in CHO-S cells is sufficient to produce predominantly agalactosylated proteins and if additional disruption of B4Gal-T4 is of any benefit with regards to N-glycan agalactosylation and decreased N-glycan heterogeneity. Additionally, we analyzed possible B4Gal-T branch preferences after combinatorial KO of B4Gal-T-isoforms. We performed CRISPR/Cas9-mediated multiplexing for all four targets followed by single cell cloning and genotype characterization via deep sequencing. Clones with different combinations of B4Gal-T-indels were expanded and further characterized with regards to cell culture performance, N-glycosylation of total secreted host cell proteins and $\mathrm{N}$-glycosylation of transiently expressed rituximab and EPO.

Targeting multiple genes in one transfection with CRISPR/Cas9 is a time saving method to generate clones with different indel-combinations in several genes. However, clones often have in-frame indels which may not disrupt the gene(s)[37]. Since increasing the number of co-transfected sgRNAs might increase the proportion of functional in-frame indels (and thereby render disrupting mutations in other genes unusable), we employed two multiplexed transfection rounds. First, we co-transfected with sgRNAs against B4Gal-T1, $\mathrm{T} 2$ and -T3 or sgRNAs against B4Gal-T1 and -T3 or sgRNAs against B4Gal-T1 and -T2. In a second round of transfection, we built up triple-KO (T1-2-3-KO and T2-3-4-KO) and quadruple KO clones (T1-2-3-4-KO) based on transfections of the T2-3-KO cell line with sgRNAs against B4Gal-T3 and -T4. Although it is faster, a limitation of this multiplexing method is that not all desired KO combinations might appear after deep sequencing of single cell clones. Ideally, the selection of clones would include at least three clones with the same gene disruptions to ensure that the resulting phenotype is not due to clonal variation upon subcloning. The efficiency of indel-generation and clone survival are therefore critical attributes when performing multiplexed experiments with CRISPR/Cas9. 
Besides influencing N-glycosylation, disrupting the four targets also influenced cell culture performance where clones with indels in B4Gal-T1, -T2 and -T3 revealed decreased growth when compared to CHO-S WT (Fig. 1). The reduced growth in clones with T1-2-3-KO and T1-2-3-4-KO could be associated to the high G0-N-glycan proportions of their secretome (Fig. 2) or be linked to clonal variation which is known to be challenging when working with CHO cells[38]. However, glycosylation plays a main role in cell-cell communication via e.g. endocytosis, receptor activation, and cell adhesion[39] and glycosylation engineering therefore might impact cultivation performance. We also report heterogeneous cell growth of clones within the generated indel combination groups. This can also be a result of clonal variation after subcloning or due to off-target effects after sgRNA and GFP_2A_Cas9 cotransfections. However, we used the sgRNA design guidelines and identical Cas9-version published in an earlier study which did not show significant off-target events[13]. While subcloning did not influence growth of the WT ctr clone, subcloning of T2-3-KO lead to decreased growth of the T2-3-K0 ctr (Fig. 1). Nonetheless, our results indicate that subcloning had no impact on secretome $\mathrm{N}$-glycosylation as the WT ctr and T2-3-KO ctr clones showed comparable N-glycan structures to their parental cell lines in the batch cultivation (Fig. 2).

In contrast to a previous study, which suggested B4Gal-T1-4 to all be active in N-glycan galactosylation[31], our results indicate that $\mathrm{B} 4 \mathrm{Gal}-\mathrm{T} 1,-\mathrm{T} 2$ and - $\mathrm{T} 3$ are the most active B4Gal-Ts in the N-glycosylation pathway of CHO-S cells and that B4Gal-T4 has very little or no contribution to galactosylation of N-glycans in CHO-S cells. The lack of N-glycosylation activity of B4Gal-T4 in our work supports another study where B4Gal-T4 was reported to be active in the galactosylation of mucin-type core 2 branching in the 0-glycosylation pathway[32]. 
Furthermore, B4Gal-T5, -T6 and -T7 (and potentially unknown B4Gal-Transferases) in sum contribute only up to $\sim 3 \% \mathrm{~N}$-glycan galactosylation of the secretome as seen in Figure 2 . The single galactose found on G1 tetra-antennary N-glycans indicates that the remaining source for N-glycan galactosylation in the quadruple-KOs can only transfer a single galactose on the N-glycan structure. However, peaks very close to the baseline could not be annotated and might harbor little amounts of structures with more than one galactose. Whether the galactosylation activity in the quadruple KO clones is carried out by B4Gal-T5, -T6, -T7 or a combination thereof has to be investigated further.

We conclude that B4Gal-T1 is the main active N-glycan B4Gal-T in clone T2-3-KO. Since the T2-3-KO clone still showed up G1, G2, G3 and G4 structures and all KO cell lines with indels in B4Gal-T1 lack G4 N-glycans, B4Gal-T1 is very likely capable of transferring galactose to all four branches. Therefore we suggest that B4Gal-T1 is the most active N-glycan processing B4Gal-T within the family of $\beta$-1,4-galactosyltransferases of CHO-S cells. The predominant activity of B4Gal-T1 in N-glycan galactosylation within our study is in line with previous work in other $\mathrm{CHO}$ cell lines[34]. Moreover, we conclude that B4Gal-T2 activity contributes to $\sim 5-10 \%$ of N-glycan galactosylation since B4Gal-T2-KO in addition to KO of B4Gal-T3 or B4Gal-T1 and -T3 increased G0 structures up to 10\% (Fig. 2).

The remaining level of rituximab galactosylation of the CHO-S derived clone T1-3-KO $(\sim 2$ $3 \%$ is comparable, yet slightly higher to another study where decreased rituximab galactosylation $(\sim 1 \%)$ was achieved by knocking out B4Gal-T1 and -T3 in CHO-K1 derived cell lines[34]. This difference in remaining N-glycan galactosylation could be due to differences in the N-glycan pathways of the cell lines used (CHO-S versus CHO-K1)[34] or due to clonal variation. Although B4gal-T3-KO lead to decreased N-glycan galactosylation activity when combined with B4Gal-T2-KO, single B4Gal-T3-KO did not decrease galactosylation at all (Fig. 2). This suggests that B4Gal-T3 has only a minor role in CHO-S N- 
glycosylation or that its disrupted N-glycan transferase function can be compensated by B4Gal-T1 and -T2 activity in the T3-KO clone.

For glycoproteins harboring tri- or tetra-antennary N-glycans, as is the case for EPO, KO of B4Gal-T1 and -T3 is not sufficient to produce mainly agalactosylated glycoproteins (Fig. 3B with 20\% EPO galactosylation in T1-3-KO), whereas rituximab expressed in clone T1-3KO resulted in only $\sim 3 \%$ galactosylated structures (Fig. 3A). Therefore, we propose that biantennary $\mathrm{N}$-glycosylated proteins as rituximab can be produced with mostly agalactosylated N-glycans after double-KO of B4Gal-T1 and -T3 but tri- and tetra-antennary N-glycosylated secretome proteins as EPO additionally need KO of B4Gal-T2 to be predominantly agalactosylated.

For transiently expressed EPO in CHO-K1 derived cells with triple-KO of B4Gal-T1, -T2 and -T3 the proportions of galactosylated $\mathrm{N}$-glycans were found to be $\sim 4 \%$ in an earlier study [34]. In our study we annotated $\sim 6 \%$ galactosylated N-glycans on transiently expressed EPO from the CHO-S derived triple-KO T1-2-3-KO A (Fig. 3C). Although these results indicate similar effects on galactosylation of EPO after disruption of two identical gene targets, deviations could be related to differences between $\mathrm{CHO}-\mathrm{K} 1$ and $\mathrm{CHO}-\mathrm{S}$ expression levels of non-targeted B4Gal-T isoforms.

We conclude that engineering cells with non-galactosylated N-glycans on a secretome level in CHO-S WT is a promising strategy towards producing G0-IgG1 and G0-EPO on a later stage. Despite the divergent gene expression levels between different CHO cell lines[40] this engineering strategy is suitable not only for CHO-K1[34] but also for CHO-S derived cell lines as utilized in our work. In the presented study the triple-KO with $\sim 1 \%$ galactosylated structures on the secretome also showed predominantly agalactosylated N-glycans on transiently expressed rituximab with only $\sim 3 \%$ galactosylated $\mathrm{N}$-glycans and on transiently expressed EPO with remaining 6\% galactosylated N-glycan structures. 
Within our triple-KO cell line T1-2-3-KO A, we also noticed a significant amount of hypermannosylated (HM) structures on transiently expressed rituximab (Fig. 3A and 3B). HM structures are a critical quality attribute within biopharmaceutical protein production[41] and can accumulate during cell culture performance. Process design and genetic engineering could be two possibilities to overcome accumulated HM structures which might represent proteins accumulated in the Golgi-situated N-glycan machinery after disrupting Golgi-residing B4Gal-T1, -T2 and -T3. This disruption might cause increased traffic and residence time of secretome proteins in the Golgi lumen without being further processed by glycosyltransferases. Recent studies displayed increased processing of Nglycans after overexpression of Mgat4 and Mgat5 which could result in decreased HM structures[42].

In N-glycan analysis of secretome, rituximab and EPO from T1-2-3-KO clones we still detected remaining galactosylated structures. Here, adding KO of B4Gal-T5, -T6 or -T7 on clone T1-2-3-KO could help to investigate their activities within the galactoslyation of $\mathrm{N}$ glycosylated proteins to e.g. remove the remaining $\sim 3 \%$ of galactosylated $\mathrm{N}$-glycans after transient rituximab expression.

The outcome of this study with the generation of different amounts of G0, G1, G2, G3 and G4 forms after disruption of the targeted transferases could be a starting point to construct a $\mathrm{N}$-glycan galactosylation model for the discussion of possible branch specificities within B4Gal-T1, -T2, -T3 and -T4 as conducted in an earlier study[31]. We suggest that the function of B4Gal-T1 includes galactosylation of all four N-glycan branches (Fig. 2), although its branch preference needs to be explored further. In clone T1-3-KO, B4Gal-T2 is suggested to be the most active B4Gal-T and only mono-galactosylated N-glycans were found within 
galactosylated structures (Fig. 2). This indicates that B4Gal-T2 N-glycosylation activity includes only the galactosylation of a single $\mathrm{N}$-glycan branch.

Studying the galactosylation levels of T1-2-KO clones in a similar approach leads to the assumption that B4Gal-T3 is at least capable of transferring galactose to up to three $\mathrm{N}$ glycan branches (Fig. 2). As presented in supplementary Figure 1, clones with T1-2-KO were identified to not produce N-glycans with decreased heterogeneity as found in the other sets of KO combinations. The origin of this heterogeneity is the increased presence of G1 Nglycan forms. However, G1 forms with further modifications can be of particular interest since they are the platform for glycoPEGylation, a method to attach polyethylene glycol (PEG) on biopharmaceuticals to increase serum half-life[43].

In summary, our study presents the necessity of disrupting the three genes, B4Gal-T1, -T2 and $-\mathrm{T} 3$, to produce not only predominantly G0 secretome proteins but also mainly agalactosylated rituximab and EPO in CHO-S cells. Further, we elucidated different N-glycan galactosylation activities within the four targeted genes where B4Gal-T1 is the most contributing enzyme to N-glycan galactosylation and involved in the galactosylation of all four N-glycan branches. Our study concludes that targeting the presented targets does only interfere with cell growth if B4Gal-T1, -T2, and -T3 are disrupted simultaneously and reveals the possibility to engineer tri- and tetra-antennary G0 N-glycans, which are naturally not produced in CHO-S WT cells (supplementary Fig. 1). We also investigated the B4Gal-T2 activity in CHO-S cells and conclude that its galactosylation activity is prevalent to one N-glycan branch while B4Gal-T4 has no N-glycan galactosylation activity and B4GalT3 can galactosylate up to three N-glycan branches. Prior engineering of secretome Nglycans in a WT cell gives rise to the flexibility of expressing several different model proteins in the engineered cell line at a later stage. Such model proteins might include already marketed antibodies or other therapeutic proteins. With our cell platform that prevalently 
glycosylates proteins with G0-N-glycans we demonstrate an alternative to galactosidase treatment of recombinant proteins to investigate further beneficial in vitro and in vivo characteristics based on tailored G0 N-glycosylation profiles.

\section{Acknowledgement}

The authors thank Sara Petersen Bjørn, Bjørn Voldborg, Johnny Arnsdorf, Yuzhou Fan and Patrice Menard for valuable guidance and support. The authors thank Karen Katrine Brøndum, Nachon Charanyanonda Petersen, Karoline Schousboe Fremming and Zulfiya Sukhova for excellent technical assistance with the FACS and MiSeq library preparation, Helle Munck Petersen for assistance with the protein purification, Anna Koza and Mads Valdemar Anderson for assistance with the MiSeq analysis. The Novo Nordisk Foundation (NNF10CC1016517) supported this work. T.A., H.F.K. and M.R.A. are receiving funding from the European Union's Horizon 2020 research and innovation program under the Marie Sklodowska-Curie grant agreement No. 642663.

\section{Author contributions}

A.H.H., M.R.A., H.F.K. and T.A. planned the experiments. T.A. performed the experimental work and wrote the manuscript. A.H.H. performed the N-glycan analysis and S.K. conducted the protein purifications. A.H.H., M.R.A., H.F.K., S.K. and G.M.L. guided the project, contributed to experimental design, and commented and corrected the manuscript.

\section{Conflict of interest}

The authors declare no financial or commercial conflict of interest. 


\section{References}

[1] Zhu, J., Mammalian cell protein expression for biopharmaceutical production. Biotechnol. Adv. 2012, 30, 1158-1170.

[2] Knaeblein, J., Modern Biopharmaceuticals: Recent Success Stories, 2013.

[3] Lai, P.H., Everett, R., Wang, F.F., Arakawa, T., et al., Structural characterization of human erythropoietin. J. Biol. Chem. 1986, 261, 3116-21.

[4] Dalziel, M., Crispin, M., Scanlan, C.N., Zitzmann, N., et al., Emerging Principles for the Therapeutic Exploitation of Glycosylation. Science (80-. ). 2014, 343, 1235681.

[5] Jennewein, M.F., Alter, G., The Immunoregulatory Roles of Antibody Glycosylation. Trends Immunol. 2017.

[6] Kanda, Y., Yamada, T., Mori, K., Okazaki, A., et al., Comparison of biological activity among nonfucosylated therapeutic IgG1 antibodies with three different N-linked Fc oligosaccharides: The high-mannose, hybrid, and complex types. Glycobiology 2007, 17, 104-118.

[7] Walsh, G., Biopharmaceutical benchmarks 2014. Nat. Biotechnol. 2014, 32, 9921000.

[8] Montacir, O., Montacir, H., Eravci, M., Springer, A., et al., Comparability study of Rituximab originator and follow-on biopharmaceutical. J. Pharm. Biomed. Anal. $2017,140,239-251$.

[9] Iida, S., Misaka, H., Inoue, M., Shibata, M., et al., Nonfucosylated therapeutic IgG1 antibody can evade the inhibitory effect of serum immunoglobulin $\mathrm{G}$ on antibodydependent cellular cytotoxicity through its high binding to Fc $\gamma$ RIIIa. Clin. Cancer Res. 2006, 12, 2879-2887.

[10] Satoh, M., Iida, S., Shitara, K., Non-fucosylated therapeutic antibodies as nextgeneration therapeutic antibodies. Expert Opin. Biol. Ther. 2006, 6, 1161-1173.

[11] Yamane-Ohnuki, N., Satoh, M., Production of therapeutic antibodies with controlled fucosylation. MAbs 2009, 1, 230-236.

[12] Malphettes, L., Freyvert, Y., Chang, J., Liu, P.Q., et al., Highly efficient deletion of FUT8 in CHO cell lines using zinc-finger nucleases yields cells that produce completely nonfucosylated antibodies. Biotechnol. Bioeng. 2010, 106, 774-783.

[13] Grav, L.M., Lee, J.S., Gerling, S., Kallehauge, T.B., et al., One-step generation of triple knockout CHO cell lines using CRISPR/Cas9 and fluorescent enrichment. Biotechnol. J. 2015, 10, 1446-1456.

[14] Louie, S., Haley, B., Marshall, B., Heidersbach, A., et al., FX knockout CHO hosts can express desired ratios of fucosylated or afucosylated antibodies with high titers and comparable product quality. Biotechnol. Bioeng. 2017, 114, 632-644.

[15] Nimmerjahn, F., Anthony, R.M., Ravetch, J. V., Agalactosylated IgG antibodies depend on cellular Fc receptors for in vivo activity. Proc. Natl. Acad. Sci. 2007, 104, 84338437.

[16] Malhotra, R., Wormald, M.R., Rudd, P.M., Fischer, P.B., et al., Glycosylation changes of IgG associated with rhematooid arthritis can activate complement via the mannosebinding protein. Nat. Med. 1995, 237-243.

[17] Wright, A., Morrison, S.L., Effect of C2-associated carbohydrate structure on Ig effector function: studies with chimeric mouse-human IgG1 antibodies in glycosylation mutants of Chinese hamster ovary cells. J Immunol 1998, 160, 33933402.

[18] Millward, T.A., Heitzmann, M., Bill, K., Längle, U., et al., Effect of constant and variable domain glycosylation on pharmacokinetics of therapeutic antibodies in mice. Biologicals 2008, 36, 41-47.

[19] Goetze, A.M., Liu, Y.D., Zhang, Z., Shah, B., et al., High-mannose glycans on the Fc region of therapeutic IgG antibodies increase serum clearance in humans. 
Glycobiology 2011, 21, 949-959.

[20] Ackerman, M.E., Crispin, M., Yu, X., Baruah, K., et al., Natural variation in Fc glycosylation of HIV-specific antibodies impacts antiviral activity. J. Clin. Invest. 2013, 123, 2183-2192.

[21] Lood, C., Allhorn, M., Lood, R., Gullstrand, B., et al., IgG glycan hydrolysis by endoglycosidase $\mathrm{S}$ diminishes the proinflammatory properties of immune complexes from patients with systemic lupus erythematosus: A possible new treatment? Arthritis Rheum. 2012, 64, 2698-2706.

[22] Dekkers, G., Plomp, R., Koeleman, C.A.M., Visser, R., et al., Multi-level glycoengineering techniques to generate IgG with defined Fc-glycans. Sci. Rep. 2016, 6, 36964.

[23] Xu, X., Nagarajan, H., Lewis, N.E., Pan, S., et al., The genomic sequence of the Chinese hamster ovary (CHO)-K1 cell line. Nat. Biotechnol. 2011, 29, 735-741.

[24] Amado, M., Almeida, R., Schwientek, T., Clausen, H., Identification and characterization of large galactosyltransferase gene families: Galactosyltransferases for all functions. Biochim. Biophys. Acta - Gen. Subj. 1999, 1473, 35-53.

[25] Furukawa, K., Sato, T., $\beta$-1, 4-galactosylation of N-glycans is a complex process. Biochim. Biophys. Acta 1999, 54-66.

[26] Ramakrishnan, B., Qasba, P.K., Crystal structure of lactose synthase reveals a large conformational change in its catalytic component, the beta1,4galactosyltransferase-I. J. Mol. Biol. 2001, 310, 205-218.

[27] Van Die, I., Van Tetering, A., Schiphorst, W.E.C.M., Sato, T., et al., The acceptor substrate specificity of human $\beta 4$-galactosyltransferase $\mathrm{V}$ indicates its potential function in 0-glycosylation. FEBS Lett. 1999, 450, 52-56.

[28] Lee, J., Sundaram, S., Shaper, N.L., Raju, T.S., et al., Chinese hamster ovary (CHO) cells may express six beta 4-galactosyltransferases (beta 4GalTs). Consequences of the loss of functional beta 4GalT-1, beta 4GalT-6, or both in CHO glycosylation mutants. J. Biol. Chem. 2001, 276, 13924-13934.

[29] Okajima, T., Yoshida, K., Kondo, T., Furukawa, K., Human Homolog of Caenorhabditis elegans sqv-3 Gene Is Galactosyltransferase I Involved in the Biosynthesis of the Glycosaminoglycan-Protein Linkage Region of Proteoglycans. J. Biol. Chem. 1999, 22915-22918.

[30] Almeida, R., Cloning and Expression of a Proteoglycan UDP-Galactose:beta -Xylose beta 1,4-Galactosyltransferase I. A SEVENTH MEMBER OF THE HUMAN beta 4GALACTOSYLTRANSFERASE GENE FAMILY. J. Biol. Chem. 1999, 274, 26165-26171.

[31] Guo, S., Sato, T., Shirane, K., Furukawa, K., Galactosylation of N-linked oligosaccharides by human -1,4-galactosyltransferases I, II, III, IV, V, and VI expressed in Sf-9 cells. Glycobiology 2001, 11, 813-820.

[32] Ujita, M., McAuliffe, J., Schwientek, T., Almeida, R., et al., Synthesis of poly-Nacetyllactosamine in core 2 branched O-glycans. J. Biol. Chem. 1998, 273, 3484334849.

[33] Asano, M., Furukawa, K., Kido, M., Matsumoto, S., et al., Growth retardation and early death of $\beta$-1,4-galactosyltransferase knockout mice with augmented proliferation and abnormal differentiation of epithelial cells. EMBO J. 1997, 16, 1850-1857.

[34] Yang, Z., Wang, S., Halim, A., Schulz, M.A., et al., Engineered CHO cells for production of diverse, homogeneous glycoproteins. Nat. Biotechnol. 2015, 33, 842-844.

[35] Slade, P.G., Hajivandi, M., Bartel, C.M., Gorfien, S.F., Identifying the CHO secretome using mucin-type O-linked glycosylation and click-chemistry.J. Proteome Res. 2012, 11, 6175-6186.

[36] Ronda, C., Pedersen, L.E., Hansen, H.G., Kallehauge, T.B., et al., Accelerating genome editing in CHO cells using CRISPR Cas9 and CRISPy, a web-based target finding tool. 
Biotechnol. Bioeng. 2014, 111, 1604-1616.

[37] Nicolas, A., Lucchetti-Miganeh, C., Yaou, R., Kaplan, J., et al., Assessment of the structural and functional impact of in-frame mutations of the DMD gene, using the tools included in the eDystrophin online database. Orphanet J Rare Dis. 2012.

[38] Vcelar S., Jadhav V., Melcher M., Auer N., et al., Karyotype variation of CHO host cell lines over time in culture characterized by chromosome counting and chromosome painting. Biotechnol. Bioeng. 2017, [Epub ahea.

[39] Ohtsubo, K., Marth, J.D., Glycosylation in Cellular Mechanisms of Health and Disease. Cell 2006, 126, 855-867.

[40] Lewis, N.E., Liu, X., Li, Y., Nagarajan, H., et al., Genomic landscapes of Chinese hamster ovary cell lines as revealed by the Cricetulus griseus draft genome. Nat. Biotechnol. 2013, 31, 759-65.

[41] Yu, M., Brown, D., Reed, C., Chung, S., et al., Production, characterization and pharmacokinetic properties of antibodies with N-linked Mannose- 5 glycans. MAbs 2012, 4, 475-487.

[42] Yin, B., Gao, Y., Chung, C. yu, Yang, S., et al., Glycoengineering of Chinese hamster ovary cells for enhanced erythropoietin N-glycan branching and sialylation. Biotechnol. Bioeng. 2015, 112, 2343-2351.

[43] Defrees, S., Wang, Z.-G., Xing, R., Scott, A.E., et al., GlycoPEGylation of recombinant therapeutic proteins produced in Escherichia coli. Glycobiology 2006, 16, 833-843. 


\begin{tabular}{|c|c|c|c|c|c|c|}
\hline \multirow{2}{*}{$\begin{array}{c}\text { parental cell } \\
\text { line }\end{array}$} & \multirow{2}{*}{$\begin{array}{c}\text { transfected with } \\
\text { sgRNA against }\end{array}$} & \multicolumn{3}{|c|}{ Target with indel size [bp] } \\
\cline { 5 - 7 } & & B4Gal-T1 & B4Gal-T2 & B4Gal-T3 & B4Gal-T4 \\
\hline \multirow{5}{*}{ CHO-S WT } & B4Gal-T1,-T2,-T3 & & & & \\
\cline { 2 - 7 } & T2-3-KO & B4Gal-T1,-T2,-T3 & & +1 & -5 & \\
\cline { 2 - 7 } & T1-3-KO & B4Gal-T1,-T3 & +1 & & +1 & \\
\cline { 2 - 7 } & T3-KO A & B4Gal-T1,-T2,-T3 & & & +1 & \\
\cline { 2 - 7 } & T3-KO B & B4Gal-T1,-T3 & & & $+1 /-10$ & \\
\cline { 2 - 7 } & T1-2-KO A & B4Gal-T1, -T2 & +1 & +1 & & \\
\cline { 2 - 7 } & T1-2-KO B & B4Gal-T1, -T2 & +1 & -19 & & \\
\cline { 2 - 7 } & T1-2-KO C & B4Gal-T1, -T2 & +1 & +1 & & \\
\cline { 2 - 7 } & T1-2-3-KO A & B4Gal-T1,-T2,-T3 & +1 & $-2 /-1$ & +1 & \\
\hline \multirow{5}{*}{ T2-3 KO } & T1-2-3-KO B & B4Gal-T1 & +1 & +1 & -5 & \\
\cline { 2 - 7 } & T1-2-3-KO C & B4Gal-T1 & +1 & +1 & -5 & \\
\cline { 2 - 7 } & T1-2-3-4-KO B & B4Gal-T1, -T4 & +1 & +1 & -5 & +1 \\
\cline { 2 - 7 } & T1-2-3-4-KO E & B4Gal-T1, -T4 & +2 & +1 & -5 & -1 \\
\cline { 2 - 7 } & T1-2-3-4-KO H & B4Gal-T1, -T4 & -1 & +1 & -5 & -1 \\
\cline { 2 - 7 } & T2-3-4-KO H & B4Gal-T1, -T4 & & +1 & -5 & -13 \\
\cline { 2 - 7 } & T2-3-4-KO K & B4Gal-T1, -T4 & & +1 & -5 & -1 \\
\cline { 2 - 7 } & T2-3-4-KO C & B4Gal-T1, -T4 & & +1 & -5 & -13 \\
\cline { 2 - 7 } & T2-3-KO ctr & B4Gal-T1 & & +1 & -5 & \\
\hline
\end{tabular}

Table 1. Overview of sgRNA/Cas 9 transfections and generated cell lines. The first round of transfections was performed with a CHO-S WT. The T2-3-KO clone was used as parental cell line for the second transfection round. Values in brackets are generated indels in bp for each target confirmed by deep sequencing. 

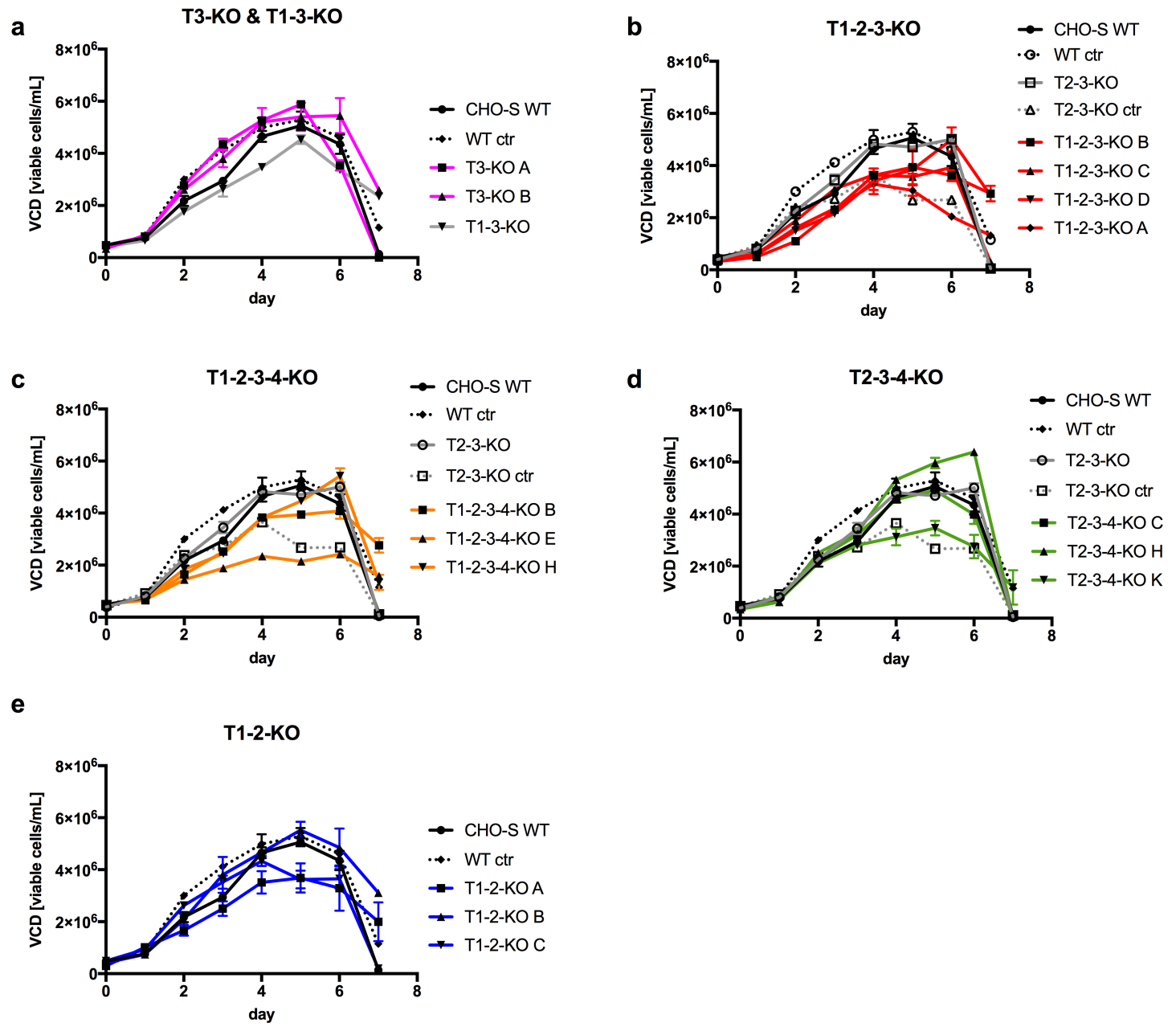

Figure 1. Growth profiles of expanded clones in batch cultivation. $30 \mathrm{~mL}$ volume batch cultivation with VCDs for the duration of seven days after sampling every $24 \mathrm{~h}(n=2)$. Seeding was performed at $3.0 \times 10^{5}$ cells $/ \mathrm{mL}$ and error bars indicate range of shake flask duplicates. (A) T3-KO \& T1-3-KO, (B) T1-2-3-KOs, (C) T1-2-3-4-KOs, (D) T2-3-KOs, (E) T12-KOs. 


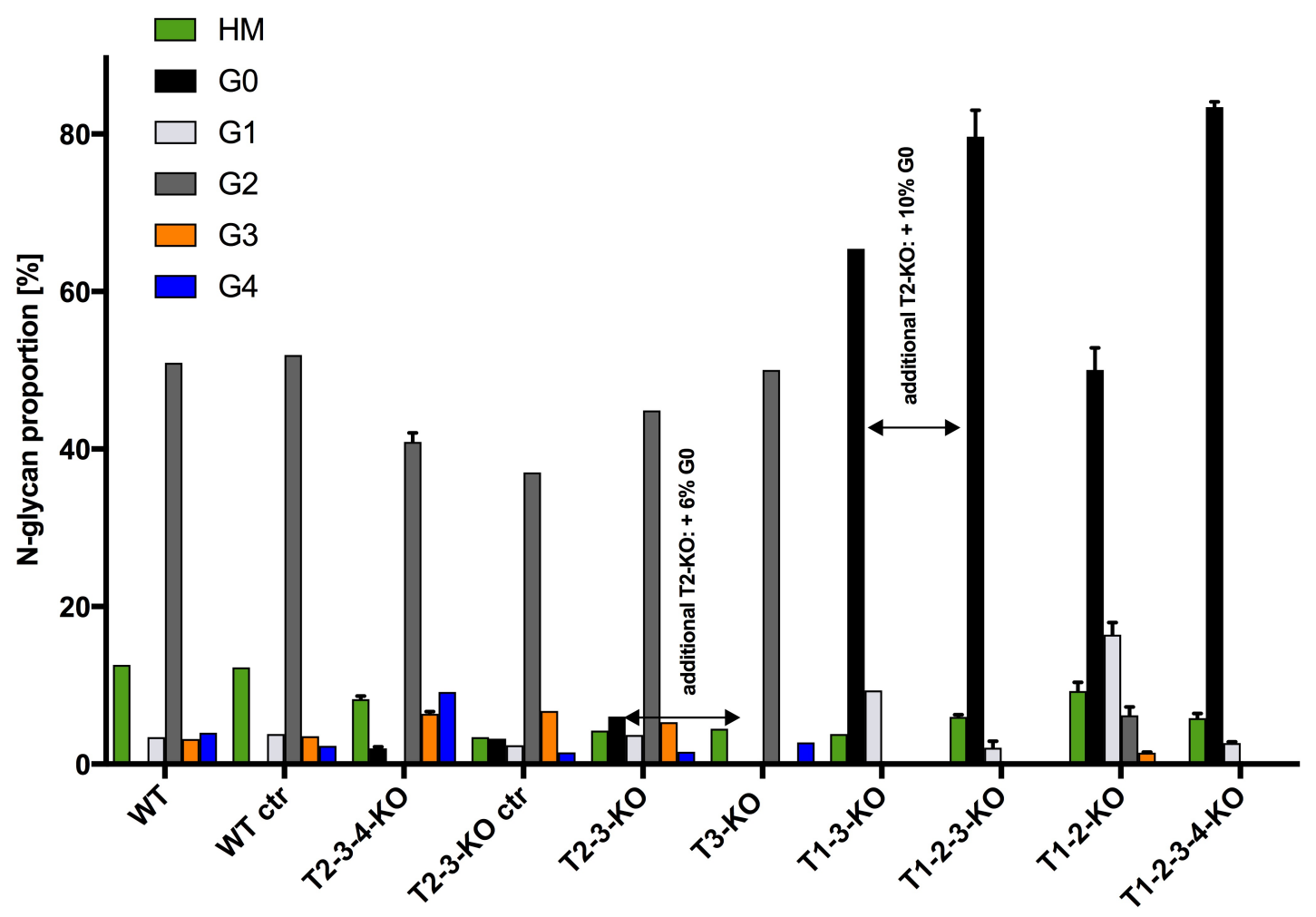

Figure 2. Secretome N-glycan profile of generated B4Gal-T-KO-clones. N-glycan secretome analysis from batch cultivation of parental cell lines and KO cell lines harvested after five days of cultivation and normalized to area under the curve (AUC) of total agalactosylated (G0), mono-galactosylated (G1), bi-galactosylated (G2), tri-galactosylated (G3), tetragalactosylated (G4) and high-mannose (HM) N-glycan peaks per cell line. Increase of G0proportion is given in \% after additional B4Gal-T2-KO in T2-3-KO and T1-2-3-KO compared to T3-KO B and T1-3-KO, respectively. Where present, error bars indicate SD of three (T12-3-KO, T2-3-4-KO and T1-2-KO) or four replicates (T1-2-3-KO). 
A

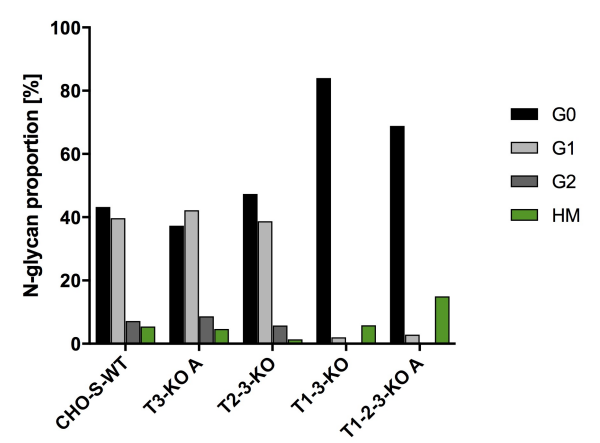

C

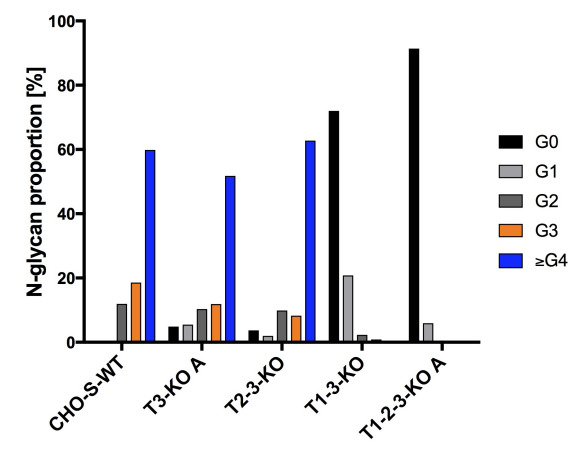

B

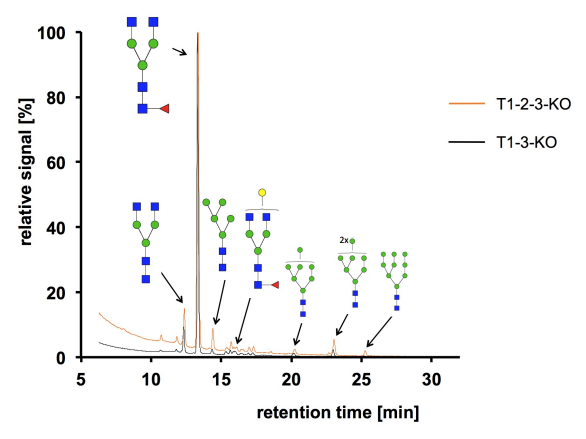

D

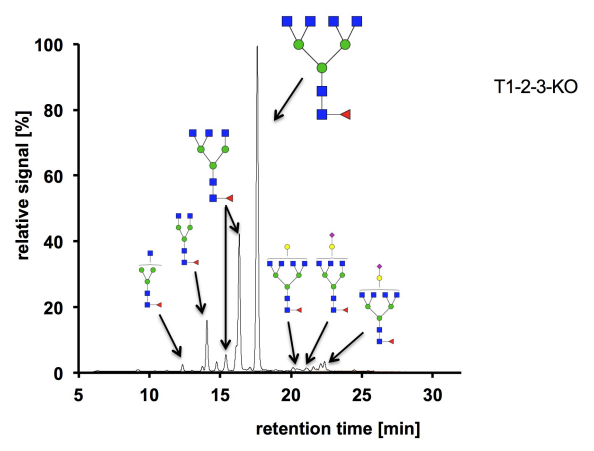

Figure 3. Rituximab and EPO N-glycosylation profiles in WT and B4Gal-T KO cell lines after transient transfection. (A) Comparison of rituximab N-glycans purified out of pooled supernatants within shake flask duplicates from CHO-S WT, T3-KO A, T2-3-KO, T1-2-3-KO A and T1-3-KO with N-glycan proportions of agalactosylated (G0), mono-galactosylated (G1), bi-galactosylated (G2) and high-mannose structures (HM) normalized to AUC of total N-glycan peaks per clone. (B) Detailed N-glycan profiles of rituximab purified out of pooled supernatants within shake flask duplicates from T1-2-3-KO A (orange line) and T1-3-KO (black line) after HPLC histogram annotation via MS. (C) Comparison of EPO N-glycans purified out of pooled supernatants within shake flask duplicates from CHO-S WT, T3-KO A, T2-3-KO, T1-2-3-KO A and T1-3-KO with N-glycan proportions of agalactosylated (G0), mono- (G1), bi- (G2), tri- (G3) and greater or equal tetra-galactosylated structures ( $\geq \mathrm{G} 4$ ) normalized to AUC of total N-glycan peaks per clone. (D) Detailed N-glycan profile of EPO purified out of pooled supernatants within shake flask duplicates from T1-2-3-KO A. 
